\title{
Enhancing immune effects of a DNA vaccine against kidney cancer using CD40L as an adjuvant
}

\author{
Guohong Jiang ${ }^{\circledR \#}$, Rixin Song ${ }^{2 \#}$, Peilong $\mathrm{Ma}^{1 *}$
}

\begin{abstract}
${ }^{1}$ Department of Nephrology, Daqing Oilfield General Hospital, 9 Zhongkang Street, Saertu District, Daqing, Heilongjiang, China, ${ }^{2}$ Department of Traditional Chinese Medicine, Daqing Oilfield General Hospital, 9 Zhongkang Street, Saertu District, Daqing, Heilongjiang, China
\end{abstract}

\begin{abstract}
The use of specific combinations of antigens and adjuvant represents a promising approach for increasing the immunogenicity of DNA vaccines. In the present study, we evaluated the immunity and antitumor effects of DNA vaccines with G250 as the target antigen in a mouse model of renal cell carcinoma. We constructed two recombinant plasmids, pVAX1-G250 and pVAX1-CD40L. The recombinant plasmids were injected into mice by intramuscular injection and electrical pulse stimulation. ELISA and ELISPOT experiments were performed to evaluate the corresponding humoral and cellular immune responses following immunization. To further investigate the antitumor potential of the DNA vaccines, we established a tumor-bearing mouse model expressing G250 target antigen. Our results showed that immunization with the combination of the two plasmids exerted the strongest anti-tumor effects. Therefore, our findings demonstrated the effectiveness of CD40L as an adjuvant for DNA vaccines and highlighted the promising use of these vaccines for the treatment of tumors.
\end{abstract}

Keywords: Kidney cancer. G250. CD40L. DNA vaccine.

\section{INTRODUCTION}

Renal cell carcinoma (RCC) is the most common malignant tumor affecting the kidneys in adults. RCC has the second highest incidence among urinary system tumors and accounts for $3 \%$ of adult systemic malignancies. The incidence of renal cell carcinoma continues to increase each year (Chander et al., 2015). Surgical treatment remains the primary treatment for renal cancer. Renal cancer is not sensitive to radiotherapy, chemotherapy, and hormone therapy, leading to generally poor prognosis. Therefore, early diagnosis and effective postoperative adjuvant therapies are particularly important for RCC (Courthod et al., 2015). Studies have shown that G250 exhibits good tumor specificity. Furthermore, $98 \%$ of primary renal cell carcinoma cases showed overexpression of the G250 antigen. In particular, the G250 antigen is overexpressed in all renal clear cell carcinoma tissues but is not expressed in normal renal tissues (Sun et al., 2014). G250 plays an important role in the survival, growth, and

\footnotetext{
*Correspondence: P. Ma. Department of Nephrology, Daqing Oilfield General Hospital, 9 Zhongkang Street, Saertu District, Daqing, Heilongjiang, 163001, China. Tel./Fax: +8613089076227. E-mail: dqytzympl@163.com

${ }^{*}$ These authors contributed equally to this work.
}

metastasis of renal tumor cells and is thus considered an ideal tumor-associated antigen (TAA). Therefore, G250 exhibits good potential for the early diagnosis, prognosis, and adjuvant therapy of RCC (Lu et al., 2014).

In recent years, DNA vaccines have been widely explored in the field of cancer treatment as a new method of immunotherapy. DNA vaccines exhibit important advantages, such as safety, high specificity, and easy industrialization and have thus become an important development direction in the immunotherapy of renal cancer (Sun et al., 2014; Lan et al., 2013). DNA vaccines are generally well tolerated and are mildly immunogenic. Therefore, research has focused on methods for improving the immunogenicity and anti-tumor effects of vaccines.

Cytokines can be used to activate immune cells, and most studies have focused on improving immunity through cytokines (Iurescia, Fioretti, Rinaldi, 2014). CD40L, also known as CD154, is a member of the tumor necrosis factor family, which are mainly expressed by activated $\mathrm{CD} 4^{+} \mathrm{T}$ lymphocytes (Gupta et al., 2014). The interaction between CD40L and CD40 can directly inhibit the growth of human breast, ovarian, cervical and bladder cancers. In addition, CD40L can induce phenotype maturation and function of DC cells, as well as enhance anti-tumor immunity by 
upregulating the expression of co-stimulatory factors and cytokines (Gupta et al., 2015).

Therefore, we reasoned that co-delivery of CD40L could serve as a feasible strategy for improving the efficacy of DNA vaccines. We aimed to enhance the effect of antitumor DNA vaccines based on G250 as target antigen in a renal carcinoma mouse model. We used CD40L as an immune adjuvant and verified its immunogenicity and antitumor effects.

\section{MATERIAL AND METHODS}

\section{Animals, cell lines, and antibodies}

BALB/c female mice (six to eight weeks old) were purchased from Vital River Laboratory Animal Technology Co., Ltd (Beijing, China). The mice were maintained in accordance with the Guide for the Care and Use of Laboratory Animal handling and experimental protocols were approved by the ethics committee of Daqing Oilfield General Hospital (Approval number: 20160805007). The murine renal carcinoma (renca) cell line was purchased from the Institute of Basic Medical Sciences, Chinese Academy of Medical Sciences (Beijing, China). Antibodies targeting the mouse CD40L (GTX75095, GeneTex Inc., USA) and human G250 (Q16790, R\&D Systems, USA) antigens were used for immunofluorescence analysis.

\section{Construction and expression of DNA vaccines}

The coding region of CD40L (M12036.1) and the coding region of G250 (GenBank: NM_001216.2) were synthesized by Invitrogen. Afterwards, the two genes were directly cloned into the eukaryotic expression plasmid vector pVAX1 (Figure 1). Two different expression plasmids were generated, namely, pVAX1- G250 and pVAX1-CD40L, corresponding to the G250 and CD40L antigens, respectively. The plasmid DNA was transformed into Escherichia coli $\mathrm{DH} 5 \alpha$ cells, purified from large-scale cultures using a TIANGEN Endo-free kit (catalog number: DP120-01, TIANGEN, Beijing, China), and suspended in sterile saline.

To evaluate the expression of these plasmids, they were transiently transfected into $293 \mathrm{~T}$ cells, and immunofluorescence analysis was performed to confirm the antigen expression of the constructs. Briefly, 293T cells were collected at $48 \mathrm{~h}$ after transfection. Cells were washed with washing buffer, treated with anti-CD40L or anti-G250 antibody, and subsequently analyzed by immunofluorescence analysis.

\section{Animal treatment groups and DNA immunization}

A total of eight BALB/c mice $(\mathrm{n}=8)$ were used for each experimental group. Mice were vaccinated with 30 $\mu \mathrm{g}$ of plasmid by intramuscular administration followed by electroporation. Vaccination was performed thrice in two-week intervals. Electroporation parameters were as follows: $120 \mathrm{~V} / \mathrm{mm}$ distance between the electrodes, 50ms pulse length, and six pulses with reversal of polarity after each pulse. The BTX 820 electroporator was used for the electroporation procedure (Hollison, USA).

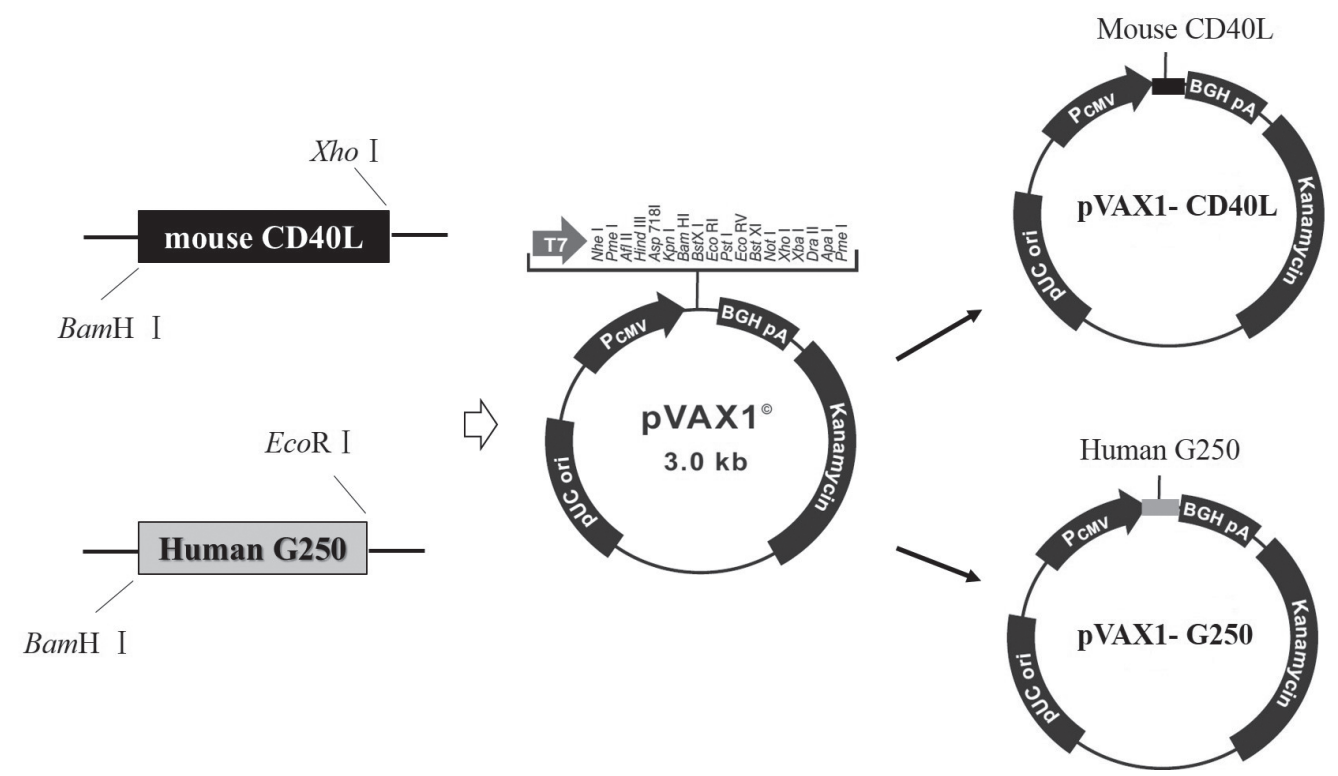

FIGURE 1 - Construction of the recombinant plasmids harboring the genes encoding G250 and CD40L. 


\section{Serum antibodies}

Anti-G250 antibodies in the sera of immunized mice were detected by enzyme linked immunosorbent assay (ELISA). Briefly, 96-well microplates were coated with $5 \mu \mathrm{g} / \mathrm{ml}$ recombinant $\mathrm{G} 250$ protein. After blocking, sera were serially diluted in PBS and incubated in the plates for $2 \mathrm{~h}$ at room temperature. Plates were washed and subsequently incubated with a 1:2000 dilution of a horseradish peroxidase (HRP)-conjugated goat antimouse IgG antibody (Bio-Sen Biological Technology, Beijing, China) at room temperature for $1 \mathrm{~h}$. The reaction was stopped with $1 \mathrm{M} \mathrm{H}_{2} \mathrm{SO}_{4}$, and the absorbance was measured at $450 \mathrm{~nm}$.

\section{Enzyme-Linked ImmunoSpot (ELISpot) assay}

Splenocytes were isolated from vaccinated groups of mice. The IFN- $\gamma$ Enzyme-Linked ImmunoSpot assay (ELISPOT) was performed according to the manufacturer's protocol (Dakewe, Beijing, China). Briefly, 96-well plates pre-coated with anti-mouse interferon (IFN)- $\gamma$ monoclonal antibody and blocked for $1 \mathrm{~h}$ at $37^{\circ} \mathrm{C}$. Freshly isolated splenocytes $\left(2 \times 10^{5}\right.$ cells/ well) from each vaccinated mouse were added to the wells and incubated with $5 \mu \mathrm{g} / \mathrm{ml}$ recombinant G250 protein for $20 \mathrm{~h}$. Assays were performed in triplicate for each treatment group.

\section{Cytokine secretion assay}

For the cytokine secretion assay, splenocytes were prepared and cultured with recombinant $\mathrm{G} 250$ protein $(5 \mu \mathrm{g} / \mathrm{mL})$ in triplicate following the same procedure as that used for the ELISPOT assays. Culture supernatants were collected after $72 \mathrm{~h}$. The concentrations of IFN- $\gamma$ and interleukin-4 (IL-4) were measured by ELISA using commercial cytokine assay kits (Dakewe, Shenzhen, China) according to the manufacturer's instructions.

\section{Tumor challenge}

Mice were vaccinated thrice with $30 \mu \mathrm{g}$ of plasmid with 14-day intervals between vaccinations. At 7 days after the final immunization, mice were subcutaneously injected with $2.0 \times 10^{5}$ renca cells in the right flank. Tumor development was monitored in individual mice every 2 to 3 days. Tumor volumes were calculated according to the following formula: $\mathrm{V}\left(\mathrm{mm}^{3}\right)=0.5 \times$ long diameters $\times$ short diameters 2, and the growth curves of the tumors were generated. After the mice were sacrificed, the tumors were removed and weighed, and the corresponding tumor growth inhibition rates were calculated.

\section{Statistical analysis}

All results were presented as means $\pm \mathrm{SD}$. Survival curves were analyzed following the Kaplan-Meier method. One-way ANOVA was performed to determine significant differences between groups. $P<0.05$ was considered statistically significant.

\section{RESULTS}

\section{Expression of DNA vaccine}

The recombinant DNA plasmids were transfected into $293 \mathrm{~T}$ cells, and expression levels of G250 and CD40L were determined by immunofluorescence analysis. Experimental results showed that both proteins were successfully expressed in mammalian cells (Figure 2).

\section{Humoral and cellular immune responses in mice}

At 14 days after the final immunization, mouse sera were collected and examined for the production of G250specific antibodies. Results revealed that immunization with DNA vaccines induced the production of G250specific antibodies (Figure 3A).

ELISPOT assays were performed to assess the ability of DNA plasmids to prime specific T cell responses. Mice were sacrificed at 14 days after the third immunization. Splenocytes were prepared and stimulated with the recombinant G250 protein. G250specific IFN- $\gamma$-producing cells were found to be induced in the immunized mice. The spot numbers in the two vaccinated groups were significantly higher than those in the empty vector $\mathrm{pVAX} 1$ group. In addition, the number of spots in the mice immunized with a combination of pVAX1-G250 and pVAX1-CD40L was higher than that in the pVAX1-G250 group (Figure 3B). The above results indicated that administration with the adjuvant plasmid harboring CD40L significantly enhanced the responses of the antigen-induced IFN- $\gamma$ producing cells from the spleen.

\section{Augmentation of Th1 and Th2 responses}

For characterization of the polarization of the immune response, IFN- $\gamma$ and IL-4 levels were measured to evaluate Th1 and Th2 cell responses, respectively. As shown in Figure 3C, Th1 cytokine (IFN- $\gamma$ ) secretion was dramatically higher in the splenocytes of mice immunized 


\section{Control blank cells}
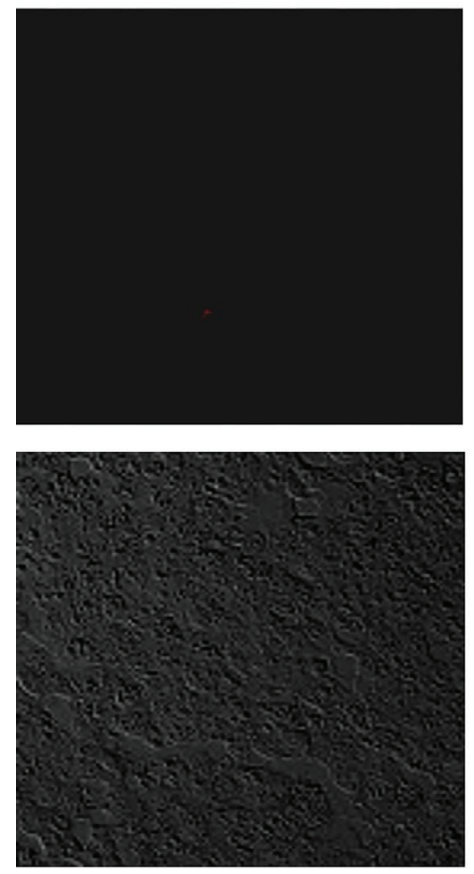

Cells transfected with pVAX1- G250
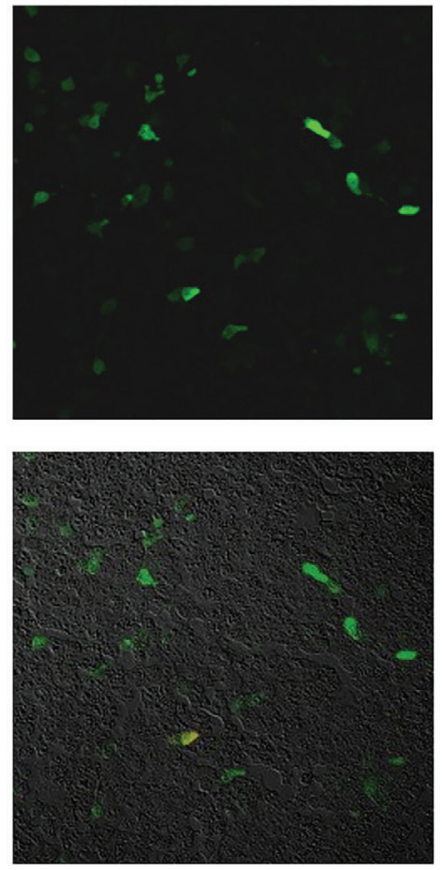

Cells transfected with pVAX1-CD40L

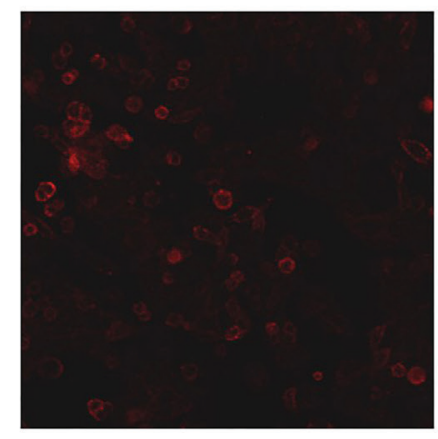

fluorescence

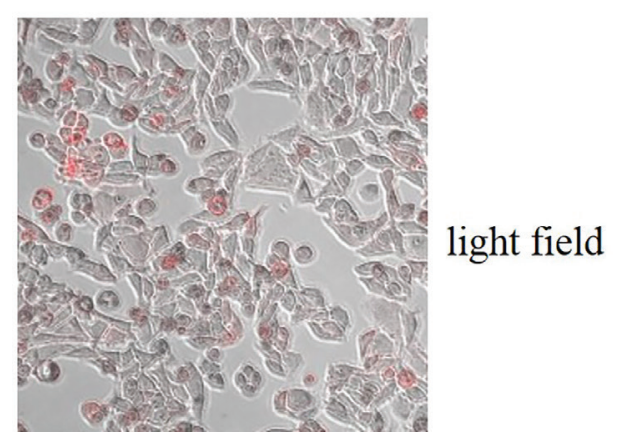

FIGURE 2 - Expression of the recombinant plasmid DNA in 293T cells by immunofluorescence analysis.

with both pVAX1-G250 and pVAX1-CD40L than in those of pVAX1-G250 group $(\mathrm{p}<0.05)$.

\section{In vivo tumor studies}

The effects of immunotherapy were demonstrated by measuring tumor size and weight. Tumor growth in mice treated with a combination of pVAX1-G250 and pVAX1CD40L or pVAX1-G250 alone was more effectively inhibited than that in the pVAX1 vector group (Fig. 4A). In addition, immunization with the combination of the pVAX1-G250 and pVAX1-CD40L vaccines was found to more effectively inhibit tumor growth than treatment with pVAX1-G250 alone. In addition, tumor weights in mice immunized with both vaccines were significantly lower than those in pVAX1-G250-immunized mice (Figure 4B).

\section{DISCUSSION}

Kidney cancer is one of the ten most common cancers affecting both men and women. In particular, kidney cancer represents $3.7 \%$ of all new cancer cases and has a worldwide incidence of over 270,000 new cases annually (Gill et al., 2016). Over the past 12 years, medical treatment for renal cell carcinoma (RCC) has transitioned from a nonspecific immune approach (cytokine era), targeted therapy against vascular endothelial growth factor (VEGF), and currently to novel immunotherapy agents. Multiple agents have been approved based on effective responses in advanced RCC patients, including the following therapies: molecules targeting vascular endothelial growth factor, platelet-derived growth factor, and related receptors; inhibitors of other targets, such as the mammalian target of rapamycin and the MET and AXL tyrosine-protein kinase receptors; and an immunecheckpoint inhibitor (Gill et al., 2016; Marenčák, Ondrušová, Ondruš, 2017).

In recent years, gene therapy as adjuvant treatment in DNA vaccination has received increasing research attention. DNA-based vaccines have several advantages over other methods of vaccination because they are generally regarded as safe and are relatively simple to produce, thereby allowing them to be quickly manufactured according to demand (Amara, Tiriveedhi, 2016). In addition, DNA plasmid vaccines are relatively stable and do not require rigorous cold-chain maintenance, making them ideal for deployment applications. Considering that cellular immunity appears to be of greater importance than humoral immunity for tumor therapy, DNA vaccines have also been traditionally considered as effective stimulators of cell-mediated immunity and are thus well suited for cancer studies (Zahm, Colluru, McNeel, 2017).

To further improve the immunogenicities of the DNA vaccines, we combined the CD40L adjuvant plasmid 
(A)

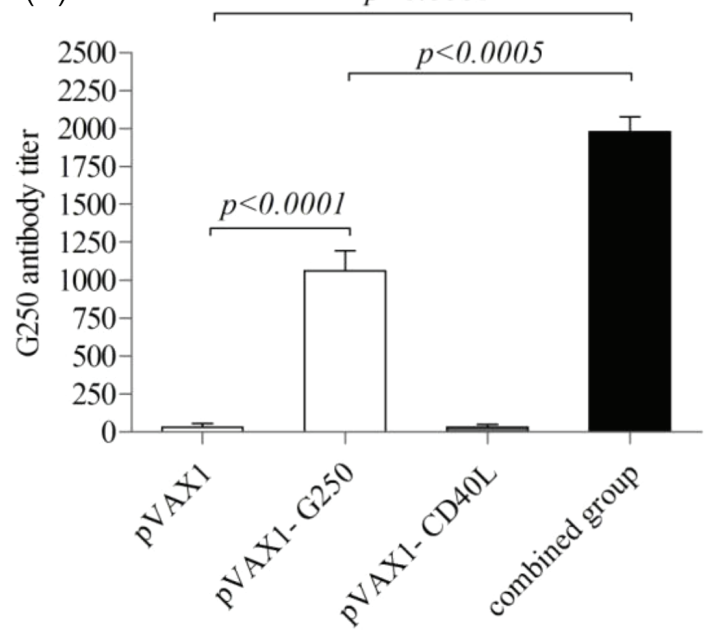

(B)

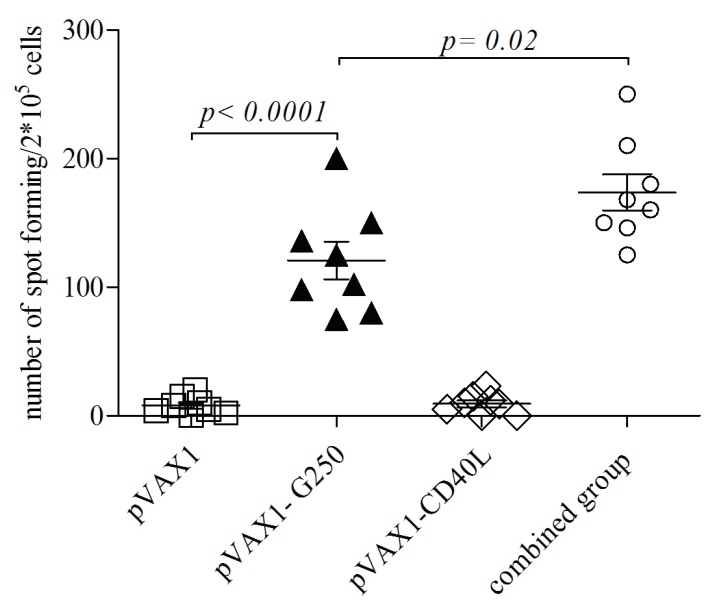

(C)

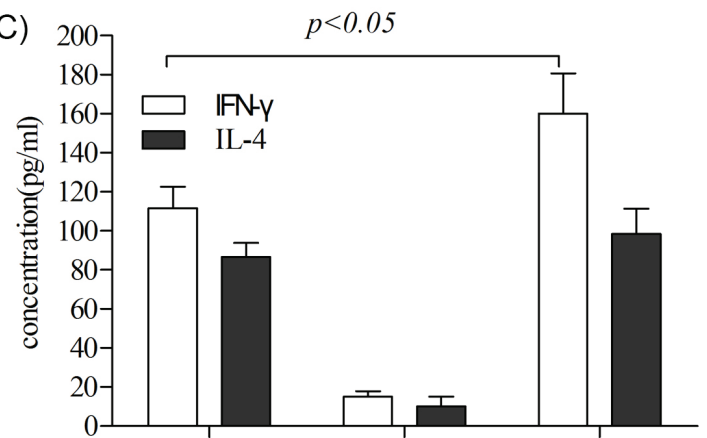<smiles>CC(C)C</smiles>

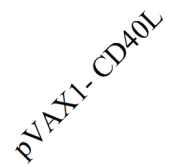

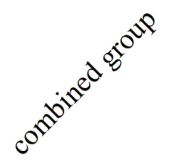

FIGURE 3 - Antibody and T cell responses induced by immunization with the recombinant DNA plasmids in mice. (A) Anti-G250 antibodies were detected by ELISA. (B) Analysis of spot frequencies of G250-specific IFN- $\gamma$-producing T cells. (C) Cytokine profiles of proliferating T cells. Data are presented as mean \pm S.D.

(A)

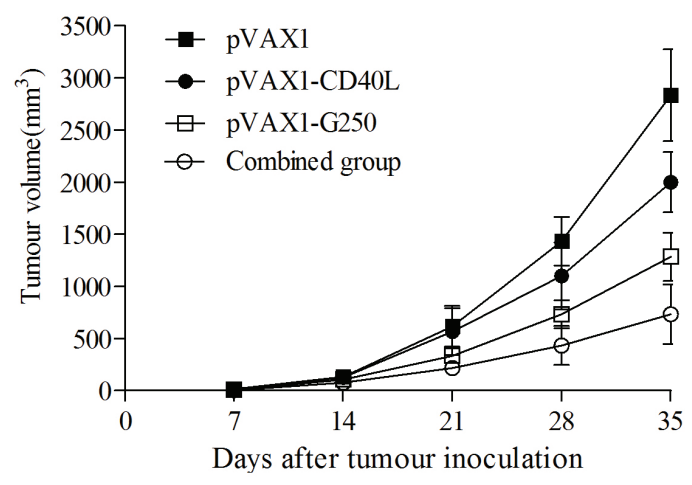

(B)

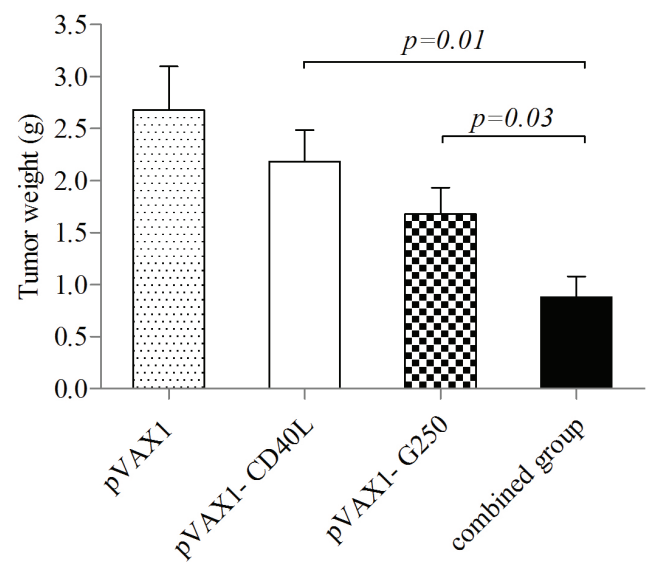

FIGURE 4 - Tumor-suppressing effects of immunization with recombinant plasmid DNA in the mouse xenograft model. (A) Growth curve of G250 ${ }^{\circledR}$ RENCA tumors. (B) Mean tumor weight. Data are presented as mean \pm S.D. Mean tumor volumes of mice immunized with the combination treatment was significantly smaller than that in mice in the other treatment groups (pVAX1, pVAX1-CD40L, or pVAX1-G250). 
with the antigen plasmid. CD40L is primarily expressed on mature CD4+ T cells. The interaction between CD40L and CD40 is important for T cell-dependent B cell activation and isotype switching. The binding between CD40L and CD40 modulates cellular immune responses by inducing the expression of costimulatory molecules that reside on antigen-presenting cells (APCs). Upregulation of costimulatory molecules lead to the activation of APCs, which in turn augment CD4+ T cell responses by increasing cytokine production, eventually leading to the activation of CD4+-dependent naive $\mathrm{CD} 8+\mathrm{T}$ cells in vivo (Levin et al., 2017; Guan et al., 2017).

Therefore, we combined G250 with CD40L to construct two plasmids, namely, pVAX1-G250 and pVAX1-CD40L, to improve the immunogenicities of the recombinant plasmids and evaluated the corresponding enhancements on anti-tumor immunity using a renal carcinoma model.

In our mouse model, immunization with a combination of the two DNA plasmids was found to be more effective in inhibiting tumor growth than immunization with either the antigen plasmid or the adjuvant plasmid alone. Analysis of the immune response showed that treatment with the combination of plasmids enhanced both the antibody and the $\mathrm{T}$ cell responses. Therefore, we hypothesized that the anti-tumor effects of the combination therapy was attributed to improved production of antigen-specific antibodies and stronger IFN- $\gamma$-mediated $\mathrm{T}$ cell responses in mice. However, the tumor growth inhibition rate was only $66 \%$ in the combination group, which indicated that the combined vaccination strategy elicited only a modest level of T cell immune response, since the cellular immune response to tumor clearance is thought to be crucial. Thus, further studies should investigate methods to further improve the potency of DNA vaccines in T cell priming, including optimization of the expression of certain $T$ cell genes and improving compatibility with other adjuvant molecules.

Experimental results revealed that vaccination with both the pVAX1- G250 and pVAX1- CD40L induced strong immune responses and tumor suppression effects and especially enhanced the G250-specific T cell response. In the xenograft model, the combination treatment led to significantly stronger inhibition of tumor growth compared with the control group (the plasmid expressing G250 alone).

\section{CONCLUSION}

In conclusion, our findings indicated that the use of CD40L as an adjuvant can effectively enhance the immunity and anti-tumor effects of a renal cell carcinoma vaccine. Our findings highlighted the promising use of CD40L for other types of tumor vaccines against tumor recurrence and metastasis.

\section{CONFLICT OF INTEREST}

The authors declare that there is no conflict of interest regarding the publication of this paper.

\section{REFERENCES}

Amara S, Tiriveedhi V. The five immune forces impacting DNA-based cancer immunotherapeutic strategy. Int J Mol Sci. 2017;18(3):pii:E650.

Chander B, Preet K, Bharti R, Deb P. Renal cell carcinoma, unclassified with unique features. J Cancer Res Ther. 2015;11(4):1027.

Courthod G, Tucci M, Di Maio M, Scagliotti GV. Papillary renal cell carcinoma: A review of the current therapeutic landscape. Crit Rev Oncol Hematol. 2015;96(1):100-112.

Gill D, Hahn AW, Sonpavde G, Agarwal N. Immunotherapy of advanced renal cell carcinoma: Current and future therapies. Hum Vaccin Immunother. 2016;12(12):2997-3004.

Guan H, Lan S, Wu J, Tang B, Xu Y. Effect of the hepatitis B virus S-ecdCD40L vaccine therapy in HBV transgenic mice: A vaccine-induced activation of antigen presenting dendritic cells. Mol Med Rep. 2017;16(5):6102-6108.

Gupta S, Termini JM, Raffa FN, Williams CA, Kornbluth RS, Stone GW. Vaccination with a fusion protein that introduces HIV-1 gag antigen into a multitrimer CD40L construct results in enhanced CD8+ T cell responses and protection from viral challenge by vaccinia-gag. J Virol. 2014;88(3):1492-1501.

Gupta S, Termini JM, Rivas Y, Otero M, Raffa FN, Bhat V, Farooq A, Stone GW. A multi-trimeric fusion of CD40L and gp100 tumor antigen activates dendritic cells and enhances survival in a B16-F10 melanoma DNA vaccine model. Vaccine. 2015;33(38):4798-4806.

Iurescia S, Fioretti D, Rinaldi M. Strategies for improving DNA vaccine performance. Methods Mol Biol. 2014;1143:21-31.

Lan KH, Liu YC, Shih YS, Tsaid CL, Yen SH, Lan KL. A DNA vaccine against cytotoxic T-lymphocyte associated antigen-4 (CTLA-4) prevents tumor growth. Biochem Biophys Res Commun. 2013;440(2):222-228. 
Levin N, Pato A, Cafri G, Eisenberg G, Peretz T, Margalit A, Lotem M, Gross G. Spontaneous activation of antigen-presenting cells by genes encoding truncated homo-oligomerizing derivatives of CD40. J Immunother. 2017;40(2):39-50.

Lu C, Li J, Xu K, Yang C, Wang J, Han C, Liu X. Fabrication of $\mathrm{mAb}$ G250-SPIO molecular magnetic resonance imaging nanoprobe for the specific detection of renal cell carcinoma in vitro. PLoS One. 2014;9(7):e101898.

Marenčák J, Ondrušová M, Ondruš D. Recent Overview of Kidney Cancer Diagnostics and Treatment. Klin Onkol. 2017;30(3):175-181.
Sun Z, Liu B, Ruan X, Liu Q. An enhanced immune response against G250, induced by a heterologous DNA prime-protein boost vaccination, using polyethyleneimine as a DNA vaccine adjuvant. Mol Med Rep. 2014;10(5):2657-2662.

Zahm CD, Colluru VT, McNeel DG. DNA vaccines for prostate cancer. Pharmacol Ther. 2017;174:27-42.

Received for publication on $19^{\text {th }}$ March 2018 Accepted for publication on $27^{\text {th }}$ June 2018 